\title{
Cytokine Profiles Associated With Worse Prognosis in a Hospitalized Peruvian COVID-19 Cohort
}

\author{
Maria J. Pons ${ }^{1 *}$, Barbara Ymaña ${ }^{1}$, Ana Mayanga-Herrera ${ }^{2}$, Yolanda Sáenz ${ }^{3}$, \\ Lydia Alvarez-Erviti ${ }^{4}$, Salyoc Tapia-Rojas ${ }^{2}$, Roxana Gamarra ${ }^{5}$, Amanda B. Blanco ${ }^{5}$, \\ Gemma Moncunill ${ }^{6}$ and Manuel F. Ugarte-Gil ${ }^{1,5}$ \\ ${ }^{1}$ Grupo Enfermedades Emergentes, Universidad Cientifica del Sur, Lima, Peru, ${ }^{2}$ Laboratorio de Cultivo Celular e \\ Inmunología, Universidad Científica del Sur, Lima, Peru, ${ }^{3}$ Área de Microbiología Molecular, Centro de Investigación \\ Biomédica de La Rioja (CIBIR), Logroño, Spain, ${ }^{4}$ Área de Neurobiología Molecular, Centro de Investigación Biomédica de La \\ Rioja (CIBIR), Logroño, Spain, ${ }^{5}$ Hospital Nacional Guillermo Almenara Irigoyen, EsSalud, Lima, Peru, 6 ISGlobal, Hospital \\ Clínic - Universitat de Barcelona, Barcelona, Spain
}

OPEN ACCESS

Edited by: Jose Miguel Rodriguez Frade, Consejo Superior de Investigaciones Cientificas (CSIC), Spain

Reviewed by: Arantzazu Alfranca, Hospital de la Princesa, Spain Jesse Huang, Karolinska Institutet (KI), Sweden Oscar Cabrera-Marante, University Hospital October 12, Spain

*Correspondence: Maria J. Pons ma.pons.cas@gmail.com

Specialty section:

This article was submitted to Cytokines and Soluble Mediators in Immunity, a section of the journal Frontiers in Immunology

Received: 27 April 2021 Accepted: 11 August 2021 Published: 01 September 2021

Citation:

Pons MJ, Ymaña B, Mayanga-Herrera A, Sáenz $Y$,

Alvarez-Erviti L, Tapia-Rojas S, Gamarra R, Blanco AB, Moncunill G and Ugarte-Gil MF (2021) Cytokine

Profiles Associated With Worse

Prognosis in a Hospitalized Peruvian COVID-19 Cohort.

Front. Immunol. 12:700921. doi: 10.3389/fimmu.2021.700921
Cytokines, chemokines and growth factors present different expression profiles related to the prognosis of COVID-19. We analyzed clinical parameters and assessed the expression of these biomarkers in patients with different disease severity in a hospitalized Peruvian cohort to determine those associated with worse prognosis. We measured anti-spike IgG antibodies by ELISA and 30 cytokines by quantitative suspension array technology in 123 sera samples. We analyzed differences between patients with moderate, severe and fatal COVID-19 by logistic regression at baseline and in longitudinal samples. Significant differences were found among the clinical parameters: hemoglobin, neutrophils, lymphocytes and C-reactive protein (CRP), creatinine and Ddimer levels. Higher anti-spike IgG antibody concentrations were associated to fatal patient outcomes. At hospitalization, IL-10, IL-6, MIP-1 $\alpha$, GM-CSF, MCP-1, IL-15, IL-5, IL1RA, TNF $\alpha$ and IL-8 levels were already increased in fatal patients' group. Meanwhile, multivariable analysis revealed that increased GM-CSF, MCP-1, IL-15, and IL-8 values were associated with fatal outcomes. Moreover, longitudinal analysis identified IL-6 and MCP-1 as the main risk factors related to mortality in hospitalized COVID-19 patients. In this Peruvian cohort we identified and validated biomarkers related to COVID-19 outcomes. Further studies are needed to identify novel criteria for stratification of SARS-CoV-2 infected patients at hospital entry.

Background: In the most severe forms of SARS-CoV-2 infection, large numbers of innate and adaptive immune cells become activated and begin to produce pro-inflammatory cytokines, establishing an exacerbated feedback loop of inflammation.

Methods: A total of 55 patients with laboratory-confirmed COVID-19 admitted to the Hospital Nacional Guillermo Almenara Irigoyen in Lima, Peru were enrolled during AugustOctober 2020. Of these, 21 had moderate disease, 24 severe diseases and 10 died. We measured 30 cytokines and chemokines by quantitative suspension array technology and anti-spike IgG antibodies using a commercial ELISA. We evaluated these parameters in peripheral blood every 2-5 days until patient discharge or death. Patient information and clinical parameters related were obtained from the respective clinical histories. 
Results: The frequency of obesity differed among the 3 groups, being most frequent in patients who died. There were also significant differences in clinical parameters: hemoglobin, segmented neutrophils, lymphocytes,C-reactive protein, creatinine and Ddimer levels. Greater anti-spike IgG antibody concentrations were associated to fatal outcomes. In univariate analyses, higher baseline concentrations of IL-6, MIP-1 $\alpha$, GMCSF, MCP-1, IL-15, IL-5, IL1RA, TNF $\alpha$, IL-8 and IL-12p70 correlated with severity, while multivariable analysis showed that increased concentrations in 4 biomarkers (GM-CSF, MCP-1, IL-15, IL-8) were associated with fatal outcomes. Longitudinal analysis showed IL-6 (hazard ratio [HR] 6.81, 95\% confidence interval [Cl] 1.6-28.7) and MCP-1 (HR 4.61, $95 \% \mathrm{Cl} 1.1-19.1)$ to be related to mortality in hospitalized COVID-19 patients.

Conclusions: Cytokine, chemokine and growth factor profiles were identified and validated related to severity and outcomes of COVID-19. Our findings may be useful to identify novel criteria for COVID-19 patient stratification at hospital entry.

Keywords: COVID-19, cytokine, prognosis, SARS-CoV-2 IgG, proinflammatory, biomarkers

\section{INTRODUCTION}

The severe acute respiratory syndrome-related coronavirus (SARS-CoV-2), which emerged in December 2019 in China, is highly pathogenic and is one of the greatest threats to public health worldwide (1). The effects of SARS-CoV-2 in infected patients are variable, with around $20 \%$ requiring hospital admission, and of these, $5 \%$ requiring ventilatory support in an intensive care unit (ICU) (2). The most serious presentations are associated with systemic inflammation (3).

The pathophysiology of COVID-19 is still under study and further research is needed to assess the causes and biomarkers of the highly variable degrees of disease progression (4). It has been described that the acute inflammatory response observed in patients with severe disease is related to an increase of some inflammatory markers such as C-reactive protein (CRP), ferritin, D-dimer, neutrophil-to-lymphocyte ratio. In addition, elevated levels of some inflammatory cytokines and chemokines have been observed in patients with severe diseases (5-8).

It is necessary to delineate the role of cytokines, chemokines and growth factors in the pathogenesis of COVID-19 across the spectrum of disease severity in different populations, ages and with and without comorbidities. Identifying prognostic biomarkers will allow prioritization of hospital resources and personalized treatment. Hence, there is great interest in determining how the severe acute respiratory syndrome alters the normal antiviral immune responses and biomarkers associated $(9,10)$. Our study aimed to identify immune-based biomarkers associated with clinical outcomes in patients hospitalized with different degrees of COVID-19 severity.

\section{MATERIAL AND METHODS}

\section{Study Population}

A cohort study was carried out including patients diagnosed with moderate or severe COVID-19 (by PCR or antibodies) hospitalized at the Hospital Nacional Guillermo Almenara Irigoyen. Patients were recruited at the time of admission to the hospital, during the period from August to October 2020. This period (mainly, August-September) coincided with the peak of active cases of the first wave of COVID-19 in Peru. It should be noted that the capacity of the available health resources, both material and human, was overstretched.

\section{Definition of Disease Severity}

Patients were stratified as having moderate or severe infection. Moderate infection was defined as requiring hospitalization and presenting an arterial oxygen partial pressure $(\mathrm{PaO} 2$ in $\mathrm{mmHg})$ to fractional inspired oxygen $\left(\mathrm{Pa} / \mathrm{FiO}_{2}\right)$ ratio greater than 100 or pulse oximetric saturation $\mathrm{Sa} / \mathrm{FiO}_{2}$ greater than 89 and $\mathrm{Pa} / \mathrm{FiO}_{2}$ less than 200 or $\mathrm{Sa} / \mathrm{FiO}_{2}$ less than 214 according to the Berlin 2012 definition of ARDS, and severe infection was defined as patients with at least one of the following conditions: having a $\mathrm{Pa} / \mathrm{FiO}_{2}$ ratio less than 100 or $\mathrm{Sa} / \mathrm{FiO}_{2}$ less than 89 ; respiratory failure requiring mechanical ventilation; shock or other organ failure requiring ICU admission.

\section{Clinical Data}

From the medical records we collected data related to age, gender, date of hospitalization, date of progression to severity and date of discharge or death, comorbidities (hypertension, coronary disease, chronic respiratory disease, diabetes, obesity and any other chronic underlying disease) as well as the pharmacological treatment (corticoids) received.

\section{Samples}

Following serological tests processed within maximum 2 hours, a total of 123 serum samples were collected (BD Vacuum Tube Vacutainer Gel SST $5 \mathrm{ml}$ ) and kept at $-20^{\circ} \mathrm{C}$ in several aliquots in the Microbiology Laboratory of the Hospital Nacional Guillermo Almenara Irigoyen. The samples were then transferred as category B biological (triple packaging) to the laboratories of the Universidad Cientifica del Sur and stored at $-80^{\circ} \mathrm{C}$ and thawed only once prior to multiplex analysis. 


\section{Quantification of Cytokines, Chemokines, and Growth Factors}

Biomarker analyses were performed using Luminex and a commercial human cytokine magnetic bead kit (Milliplex Human Cytokine/Chemokine MAGNETIC BEAD Premixed 30 Plex Kit). This kit measures the concentrations $(\mathrm{pg} / \mathrm{mL})$ of the following cytokines, chemokines and growth factors: epidermal growth factor (EGF), eotaxin (CCL11), granulocyte colony stimulating factor (G-CSF), granulocyte macrophage colony stimulating factor (GM-CSF), interferon (IFN)- $\alpha$, IFN$\gamma$, interleukin (IL) -1 $\alpha, 1 \mathrm{RA}$, IL-1 $\beta$, IL-2, IL-12, IL-3, IL-4, IL-5, IL6, IL-7, IL-8, IL-10, IL-12 (p40/p70), IL-13, IL-15, IL-17, IP-10, monocyte chemoattractant protein-1 (MCP-1), macrophage inflammatory protein (MIP)-1 $\alpha$, MIP-1 $\beta$, RANTES, tumor necrosis factor (TNF $\alpha$ ), TNF $\beta$ and vascular endothelial growth factor (VEGF). Fifty $\mu$ of all the serum samples were tested according to the manufacturer's instructions in individual replicates distributed on two plates. Each plate included 8 dilutions of a standard for standard curves provided by the vendor with a known concentration of each analyte. Two blank controls and three positive controls in duplicates of high, medium and low concentrations prepared from a reference sample were also included on each plate for quality control and quality assurance purposes. The samples were measured using MAGPIX ${ }^{\circledR}$ and the results were analyzed with xPONENT 3.1 software.

\section{Measurement of Antibodies Anti-SARS-CoV-2}

Anti-spike SARS-Cov-2 protein IgG was evaluated by a semiquantitative commercial kit (EuroImmune), according to manufacturer's instructions. The results were evaluated by calculating a ratio of the absorbance of the patient sample to the absorbance of the calibrator ( $>1$ results were considered positive). The cut-off was defined following the manufacturer's recommendations. The absorbance values were read on the Biotek Synergy LX Multi-Mode Reader (Agilent).

\section{Statistical Analysis}

We performed a descriptive analysis summarizing each categorical variable with the absolute and relative frequencies and each numerical variable with the mean and standard deviation. We normalized cytokine concentration with its logarithm with base 10. Then, we compared each descriptor by outcome (moderate, severe, and death) using the chi square test for proportion comparisons and the Kruskal-Wallis test with Dunn's test for levels comparisons. Correlations between clinical parameters, immunoglobulin levels, and cytokine, chemokine and growth factor concentrations were assessed by Spearman tests. To identify clusters of biomarkers associated with the severity group, we performed Parial Least Squares - Discriminant Analysis (PLS-DA). The analyses of prognostic factors were performed using logistic regression. We performed two sets of analysis. First, we assessed the predictability of death using only the patient descriptors at baseline. This analysis included a Spearman correlation analysis and regression analysis using generalized linear models with a Poisson distribution, link log, and robust error variance. Second, we performed longitudinal and association analysis between biomarkers with mortality using Cox regression models. Hazard ratios (HR) were therefore estimated as the magnitude of the association of interest. All models were fitted using the forward method and the Akaike Information Criterion. Each parameter was estimated using a confidence interval of $95 \%$ (95\% CI). All the analyses were performed using STATA MP version 14.0 (Stata Corp., College Station, TX) and all the graphics were elaborated using $\mathrm{R}$ studio version R-4.0.2.

\section{Ethical Aspects}

This project was approved by the Institutional Ethics Committee of the Universidad Cientifica del Sur and the Research Ethics Committee for COVID-19 of IETSI-EsSalud (42-IETSIESSALUD-2020).

In order to avoid exposing workers to infectious areas and the need to obtain additional samples from patients, leftover serum samples taken for diagnostic purposes during hospitalization were analyzed. No additional samples were taken from the patient, so no additional inconvenience was generated.

\section{RESULTS}

\section{Baseline Clinical Characteristics of COVID-19 Hospitalized Patients}

Fifty-five patients hospitalized for COVID-19 disease were included: 21 (38.2\%) in the moderate disease group, $24(43.6 \%)$ in the severe group and 10 in the deceased group (18.2\%), according to maximum state category reached during the follow-up period. The mean age of the patients was 54, 51 and 52 years, respectively, with no differences in the median age and sex among the 3 groups $(\mathrm{p}=0.360, \mathrm{p}=0.244$, respectively). Regarding comorbidities, 24 patients (43.6\%) had no comorbidities and $31(56.4 \%)$ had at least one comorbidity, including $15(27.3 \%)$ patients with obesity, 12 (21.8\%) with hypertension, and 7 (12.7\%) with diabetes. Only obesity presented differences between severity groups, being more frequent in the deceased group $(\mathrm{p}=0.002)$ (Table 1). Moreover, two patients (3.6\%) had obesity, hypertension, and diabetes and two $(3.6 \%)$ patients had both obesity and hypertension. Finally, only 1 patient (1.9\%) had hypertension and diabetes and 1 patient $(1.9 \%)$ presented obesity and diabetes. The presence of other comorbidities such as chronic kidney disease, chronic obstructive pulmonary disease (COPD), cardiovascular disease (CVD) and gout is shown in Table 1. No patients with liver disease or deep vein thrombosis (DVT) were reported. All hospitalized patients received corticosteroid treatment from the beginning of their stay.

\section{Clinical Factors and Laboratory Tests at Baseline Associated With Differential Mortality}

Regarding hematological parameters, there were significant differences in hemoglobin levels at baseline, being lower in the 
TABLE 1 | Characteristics of the study population.

\begin{tabular}{|c|c|c|c|c|}
\hline & Moderate & Severe & Deaths & p-value \\
\hline Overall, (n,\%) & $21(38.2)$ & $24(43.6)$ & $10(18.2)$ & \\
\hline Men (n,\%) & 17 (80.9) & $22(91.7)$ & $10(100)$ & 0.244 \\
\hline Age, mean (sd), yr & $54.6(16)$ & $51.1(11.2)$ & $52(14.3)$ & 0.360 \\
\hline Hospital discharge, mean (sd), days & $11.9(8.2)$ & $12.9(7.1)$ & $13.5(7.1)$ & 0.898 \\
\hline \multicolumn{5}{|l|}{ Comorbidities } \\
\hline None, n (\%) & $11(52.4)$ & $12(50.0)$ & $1(10)$ & 0.059 \\
\hline Obesity, n (\%) & $2(9.5)$ & $6(25.0)$ & $7(70)$ & 0.002 \\
\hline Diabetes, n (\%) & $4(19.1)$ & $1(4.2)$ & $2(20.5)$ & 0.245 \\
\hline Hypertension, n (\%) & $4(19.1)$ & $5(20.8)$ & $3(30)$ & 0.779 \\
\hline CKD, n (\%) & $0(0)$ & $1(4.2)$ & $0(0)$ & 0.518 \\
\hline COPD, n (\%) & $1(4.8)$ & $0(0)$ & $2(20)$ & 0.064 \\
\hline CVD, n (\%) & $0(0)$ & $0(0)$ & $1(10)$ & 0.101 \\
\hline DVT, n (\%) & $0(0)$ & $0(0)$ & $0(0)$ & NA \\
\hline Gout, n (\%) & $1(4.8)$ & $0(0)$ & $0(0)$ & 0.438 \\
\hline Other diseases & $4(19.1)$ & $1(4.2)$ & $0(0)$ & 0.121 \\
\hline \multicolumn{5}{|l|}{ Hemogram } \\
\hline Hemoglobin, mean (sd), g/dL & $14.3(1.7)$ & $14.6(1.4)$ & $12.7(1.2)$ & 0.004 \\
\hline Leukocytes, mean (sd), $10^{9} / \mathrm{L}$ & $10.4(4.6)$ & $10.6(3.9)$ & $13.1(3.0)$ & 0.208 \\
\hline Basophiles, mean (sd) & $2.8(3.4)$ & $1.9(1.5)$ & $2(1.5)$ & 0.602 \\
\hline Segmented neutrophils, mean (sd) & $81.1(12.0)$ & $81.2(6.2)$ & $89.3(4.6)$ & 0.037 \\
\hline Lymphocytes, mean (sd) & $13.3(10.7)$ & $12.5(5.7)$ & $6.3(4.1)$ & 0.001 \\
\hline Neutrophils, mean (sd), (total) & $8.9(4.9)$ & $8.8(3.6)$ & $11.8(3.1)$ & 0.124 \\
\hline lymphocytes, mean (sd), (total) & $1.1(0.7)$ & $1.2(0.5)$ & $0.8(0.5)$ & 0.146 \\
\hline CRP, mean (sd), mg/ml & 125 (109) & $57(67)$ & $179(121)$ & 0.007 \\
\hline Platelets, mean (sd), count/ul & 3336857 (94290) & 363000 (113701) & 274750 (41709) & 0.101 \\
\hline Creatinine, mean (sd), mg/dl & $0.8(0.6)$ & $0.8(0.5)$ & $1.8(1.9)$ & 0.024 \\
\hline D-Dimer, mean (sd), ug/ml & $0.9(0.7)$ & $0.8(0.9)$ & $3.0(1.6)$ & $<0.001$ \\
\hline Prothrombin Time, mean (sd), sec. & $11.2(0.8)$ & $11.3(0.8)$ & $10.8(0.5)$ & 0.347 \\
\hline TTPA, mean (sd), sec. & $32.2(4.3)$ & $31.3(4.8)$ & $34.8(6.4)$ & 0.309 \\
\hline $\mathrm{SaFiO}_{2}$, mean (sd) & $242.7(98.1)$ & $240.1(100.6)$ & $155.7(79.2)$ & 0.047 \\
\hline Ferritin, mean (sd), ug/L & 759.1 (389) & $968.3(630)$ & $1247(754)$ & 0.103 \\
\hline
\end{tabular}

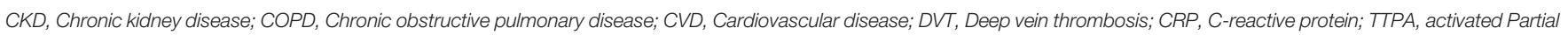
Thromboplastin Time; $P$-value $<0.05$ were statistical significance and values are marked with bold numbers.

deceased group $(12.7 ; \pm 1.2)(\mathrm{p}=0.004)$ (Table 1$)$. In addition, lymphocyte levels were also lower in the severe and deceased groups ( $\mathrm{p}=0.001)$, while the more severe groups presented higher mean segmented neutrophil values $(\mathrm{p}=0.037)$. No significant differences were observed between the 3 groups for the remaining hematological parameters.

With respect to analytical parameters, significant differences were observed in CRP, D-dimer and creatinine values between the moderate, severe and deceased groups $(p=0.007, p<0.001$ and 0.020 , respectively), with the deceased group presenting the highest mean values. There were differences between groups in relation to the $\mathrm{Sa} / \mathrm{FiO}_{2}$ ratio, with the deceased group also showing lower values than the other two groups (Table 1).

\section{Anti-Spike IgG Antibodies of SARS-CoV-2 at Baseline}

Most patients had detectable antibodies against the spike 1 protein of SARS-CoV-2, except for 7 patients (12.7\%). As shown in Figure 1, there were significant differences among the 3 groups $(\mathrm{p}=0.013)$, with higher levels observed in the deceased group compared to the moderate and severe group.

\section{Cytokine Levels in the Moderate, Severe, and Deceased Groups at Baseline}

Significant differences were found between groups for, IL-6 $(p=0.0075)$, MIP-1 $\alpha(p=0.017)$, GM-CSF $(p=0.001)$, MCP-1 $(p<0.001)$, IL-15 $(p=0.005), \operatorname{IFN} \alpha(p=0.002), \quad I L-1 R A$ $(p=0.012)$, TNF $\alpha(p=0.030)$, IP-10 $(p=0.014)$, IL-8 $(p<0.001)$ and IL-12p70 ( $\mathrm{p}=0.045)$ levels. All the biomarkers presented higher values in the deceased group than the moderate and/or severe group (Figure 2).

\section{Cytokine and Antibodies Correlations at Baseline}

We observed a significantly high correlation between biomarker levels in COVID-19 patients (Figure 3). Positive correlations were found between pro-inflammatory and anti-inflammatory biomarkers, while negative correlations were only observed for RANTES and IL-8. The biomarkers EGF, VEGF, eotaxin, MIP$1 \beta$, and TNF- $\beta$ did not show any significant correlations with the other biomarkers.

There was a positive and moderate correlation between antispike IgG and IL-8 $(\rho=0.31 ; p=0.02)$, while G-CSF $(\rho=-0.33$; $p=0.013)$, IFN- $\gamma(\rho=-0.47 ; p<0.001)$ and IP-10 $(\rho=-0.38$; $\mathrm{p}=0.004$ ) showed an inverse correlation (Figure 4). 


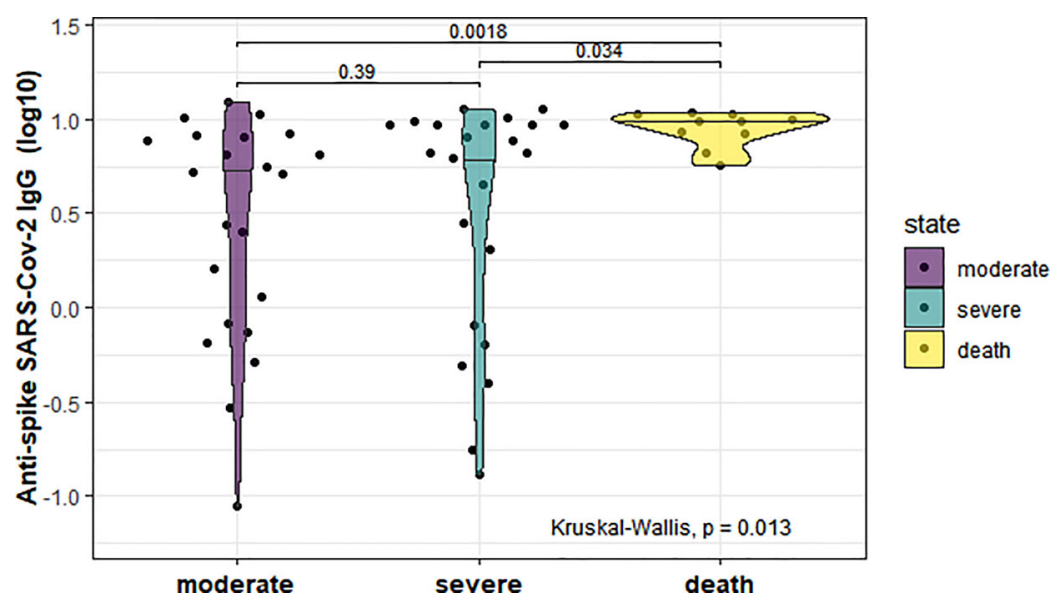

FIGURE 1 | Serum anti-spike IgG concentrations in the three study groups (moderate, severe and deceased) of hospitalized COVID-19 patients at baseline. The median is shown in different violin groups of biomarkers. P-values were computed by the Kruskal-Wallis test and a post-hoc analysis by Dunn's test.

\section{Multi-Biomarker Associations at Baseline}

In the PLS-DA analysis we identified several components that were negatively associated with the severe COVID-19 group. Figure $5 \mathrm{~A}$ shows the most predictive components (components $1,2)$. The biomarkers contributing most to the components were those with higher loadings in the different components (high relevant biomarkers were considered with loadings $<-0.50$ and very high relevant biomarkers were considered with loadings $<-0.75)$. In component 1 , the most relevant biomarkers were IL10, IL-13, IL-6, IL-12, MIP-1 $\alpha$, MCP-1, IFN $\alpha$, IL1RA, IP-10, IL4 , IL-8, and IL-12p70 all of which had negative loadings. Additionally, the biomarkers with the highest relevance were IL-1 $\beta$, G-CSF, GM-CSF, IL-15, IL-2 and IL- $1 \alpha$, also with negative loadings (Figure 5B).

\section{Biomarkers at Baseline Associated With Mortality in Bivariate and Multivariable Regression Analysis}

We performed bivariate regression analysis for each of the 30 cytokines, chemokines, growth factors and IgG antibodies to identify those associated with mortality. Increased levels of 11 biomarkers at baseline were associated with increased mortality. These biomarkers included anti-spike IgG levels (IRRc=188.57), biomarkers associated with monocyte/macrophage activation and/or activation of NF-KB MCP-1/CCL2 (IRRc=7.09) and IL$6(\mathrm{IRR} c=2.38)$, as well as proinflammatory chemokine MIP- $1 \alpha$ (CXCL10) $($ IRRc=10.88) and IL-8, IRRc=7.31 and stimulatory type proinflammatory Th1 cytokines, TNF $\alpha($ IRRc $=2.68)$ and IL15 (IRRc=9.99); the Th2 mediator IL-5 IRRc=(5.43), and antiinflammatory cytokine IL-10 $($ IRRc $=1.61)$ and IL-1RA $($ IRRc $=3.00) \quad($ Table 2$)$. The growth factor GM-CSF $($ IRRc $=188.57)$ was also associated with death (Table 2). In the multivariable analysis, the biomarkers GM-CSF (IRRa $=0.003$ ), MCP-1 (IRRa=17.99) and IL-8 (IRRa=3.80) and anti-spike IgG $(\mathrm{IRRa}=16.34)$ were significantly associated with mortality (Table 2$)$.

\section{Longitudinal Analysis and Association Between Biomarkers and Mortality}

In the longitudinal bivariate analysis, we observed a significant association between mortality and 15 biomarkers: IL- $1 \alpha$ $(\mathrm{p}=0.001), I L-1 \beta(\mathrm{p}=0.050), I L-10 \quad(\mathrm{p}=0.012), I L-13 \quad(\mathrm{p}=0.007)$, IL-6 $(\mathrm{p}<0.001)$, IL-15 $(\mathrm{p}<0.001)$, IL-5 $(\mathrm{p}=0.008)$, IL1RA $(\mathrm{p}=0.010), I L-2(\mathrm{p}=0.022), I L-8 \quad(\mathrm{p}=0.007), I P-10(\mathrm{p}=0.004), \mathrm{G}-$ CSF $(\mathrm{p}=0.015)$, GM-CSF $(\mathrm{p}=0.006)$, MCP-1 $(\mathrm{p}=0.001)$, and $\operatorname{TNF} \beta$ ( $\mathrm{p}=0.045)$. However, in the Cox regression multivariable analysis the main predictors of death by COVID-19 in hospitalized patients were IL-6 (HR =6.81; 95\% CI: 1.6-28.7) and MCP-1 (HR $=4.61 ; 95 \%$ CI: 1.1-19.1) (Figure 6).

\section{DISCUSSION}

We have profiled the cytokine/chemokine/growth factor and clinical parameters associated with COVID-19 fatal outcome in Peruvian hospitalized patients. Clinical parameters associated with death were lower hemoglobin and lymphocytes, and higher segmented neutrophils, CRP, creatinine and D-dimer levels. Higher concentration of IgG anti-spike-1 antibodies patients were also associated with mortality, as well as higher IL-10, IL6, MIP-1 $\alpha$, GM-CSF, MCP-1, IL-15, IL-5, IL-1RA, TNF $\alpha$ and IL-8 in bivariable regression analysis and GM-CSF, MCP-1, IL15 , IL-8 in multivariable regression analysis. Longitudinal analysis showed higher concentrations of IL- 6 and MCP-1 associated with higher risk of mortality in COVID-19 hospitalized patients. The mean age of 53 years of our study participants was lower than that of other studies in which older age was identified as a predictor of poor outcomes and were mainly composed of men, with male sex also being associated with fatal outcomes (11). Comorbidities have been described as playing an important role in prognosis. We found a high frequency of obesity $(70 \%)$ in the deceased group. Of note, a 

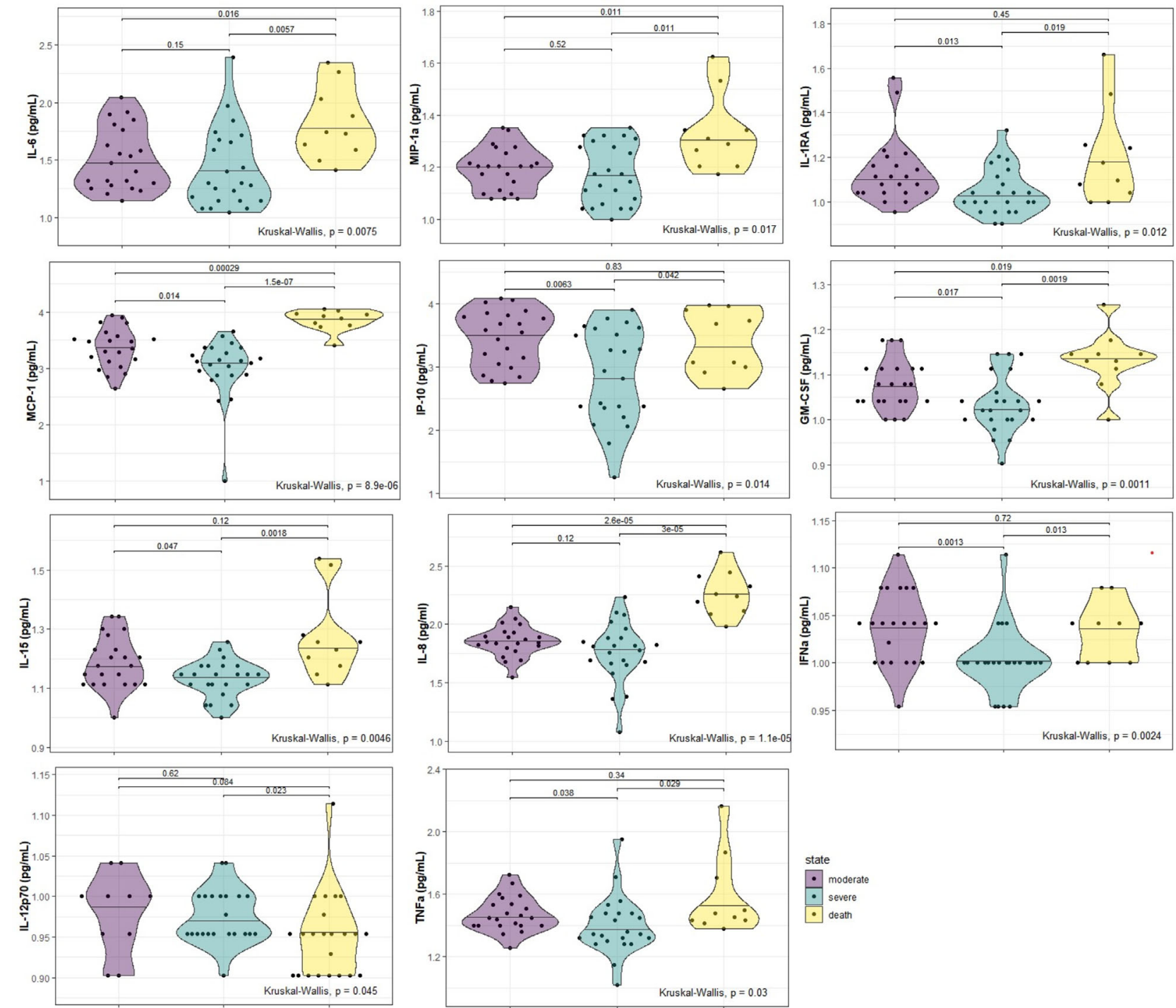

\begin{tabular}{l} 
state \\
\hline$-\dot{0}^{\text {moderate }}$ \\
\hline
\end{tabular}

\begin{tabular}{l}
- \\
\hline \\
\hline
\end{tabular}

FIGURE 2 | Serum biomarkers with significant differences in the three study groups (moderate, severe and deceased) of hospitalized COVID-19 patients at baseline. P-values were computed by the Kruskal-Wallis test and a post-hoc analysis by Dunn's test. 


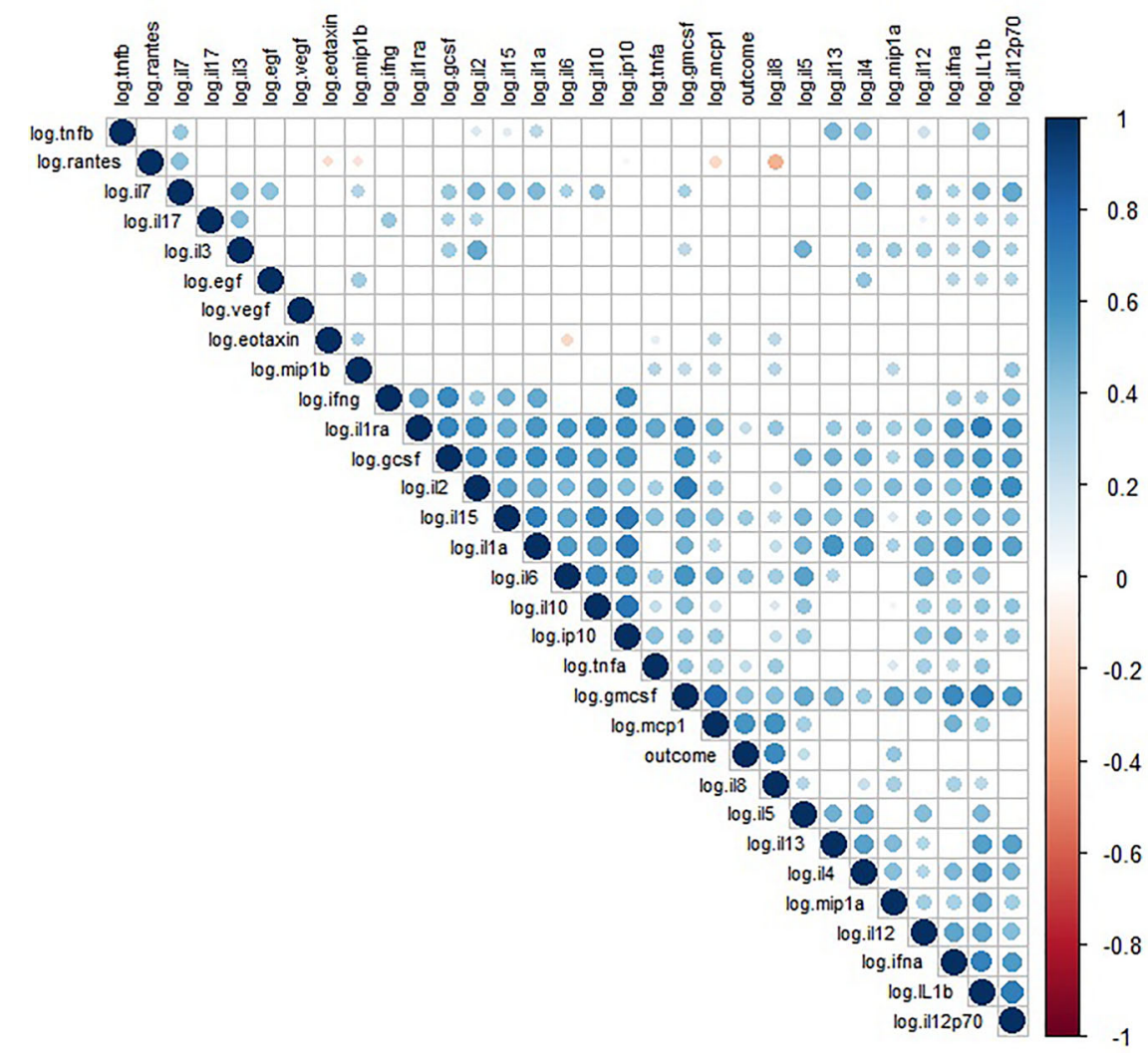

FIGURE 3 | Spearman correlation matrix between biomarkers concentrations. Only significant correlations are shown ( $p>0.05)$. The outcome in this analysis is death.
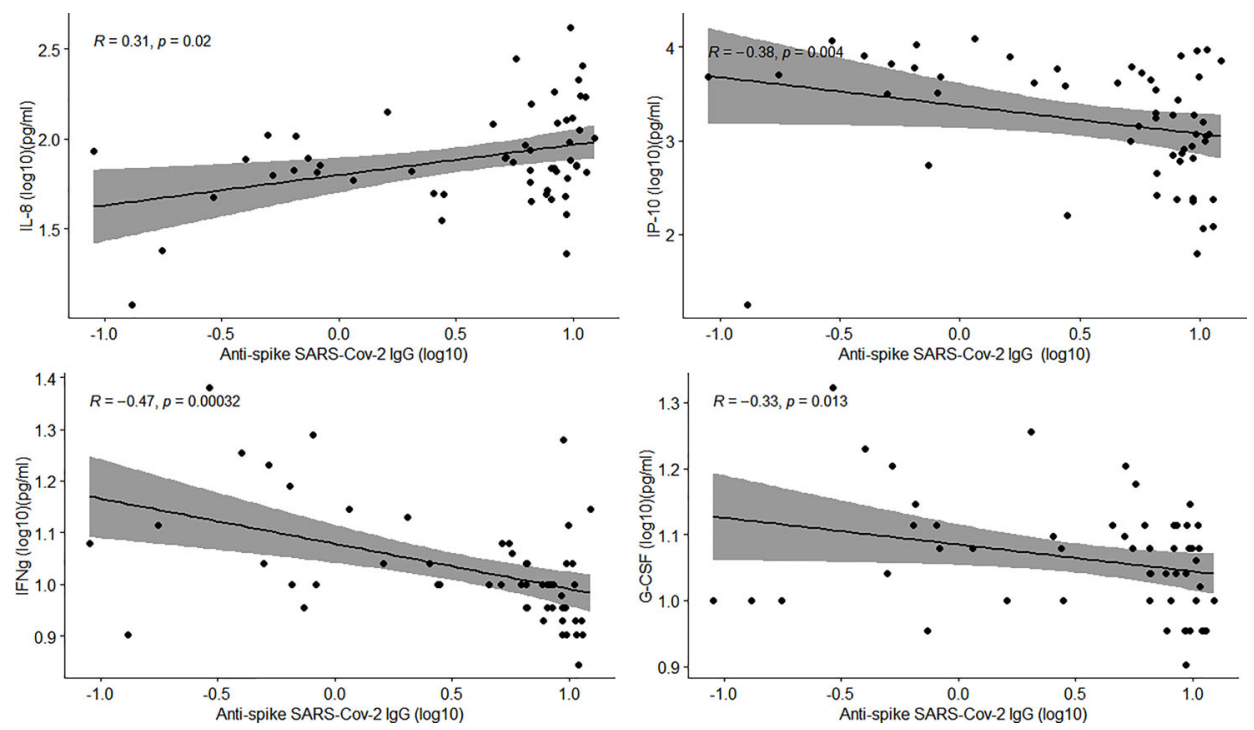

FIGURE 4 | Correlation of anti-spike IgG antibody levels and biomarker concentrations in COVID-19 patients. Scatter plots show statistically significant correlations between biomarkers and antibody levels. Rho value and p-vale were computed by Spearman correlations. The shaded area shows the $95 \%$ confidence interval. 
A

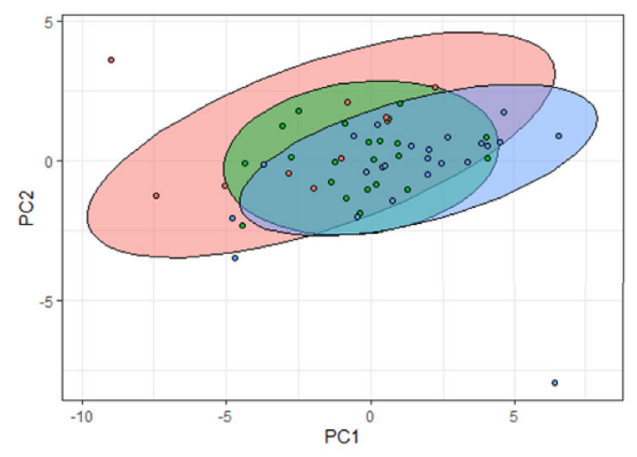

B

\begin{tabular}{|c|c|c|}
\hline & PC1 & PC2 \\
\hline IL-1 $1 \beta$ & -0.81 & -0.23 \\
\hline G-CSF & -0.75 & -0.18 \\
\hline IL-10 & -0.66 & 0.13 \\
\hline IL-13 & -0.55 & -0.25 \\
\hline IL-6 & -0.6 & -0.04 \\
\hline IL-12 & -0.62 & -0.28 \\
\hline RANTES & -0.31 & 0.51 \\
\hline EOTAXIN & -0.08 & 0.66 \\
\hline [L-17 & -0.38 & -0.38 \\
\hline MIP-1 $1 \alpha$ & -0.55 & -0.07 \\
\hline GM-CSF & -0.82 & 0.09 \\
\hline MIP- $1 \beta$ & -0.27 & 0.58 \\
\hline MCP-1 & -0.55 & 0.6 \\
\hline |L-15 & -0.8 & 0.15 \\
\hline EGF & -0.23 & 0.14 \\
\hline IL-5 & -0.48 & -0.22 \\
\hline VEGF & -0.1 & 0.01 \\
\hline IFNY & -0.41 & -0.04 \\
\hline IFNa & -0.7 & -0.05 \\
\hline IL-1RA & -0.77 & 0.21 \\
\hline TNF $\alpha$ & -0.5 & 0.52 \\
\hline LL-2 & -0.79 & -0.21 \\
\hline IL-7 & -0.49 & -0.09 \\
\hline |P-10 & -0.63 & 0.2 \\
\hline IL-4 & -0.61 & -0.23 \\
\hline IL-8 & -0.54 & 0.47 \\
\hline |L12p70 & -0.68 & -0.04 \\
\hline IL-1 $\alpha$ & -0.76 & -0.24 \\
\hline IL-3 & -0.39 & -0.43 \\
\hline INF $\beta$ & -0.4 & -0.21 \\
\hline
\end{tabular}

FIGURE 5 | Partial Least-Squares Discriminant Analysis (PLS-DA). (A) Graph of PLS-DA plots representing each sample (dots) with respect to the first 2 PLS-DA components according to severity group (moderate, severe, deceased). (B) Graph of marker loading to the 2 components of the PLS-DA. Principal component (PC).

TABLE 2 | Bivariate and Multivariable regression analysis of biomarkers associated with death in hospitalized COVID-19 male patients.

\begin{tabular}{|c|c|c|c|c|}
\hline & \multicolumn{2}{|c|}{ Bivariate } & \multicolumn{2}{|c|}{ Multivariable } \\
\hline & IRR & $95 \% \mathrm{Cl}$ & IRRa & $95 \% \mathrm{Cl}$ \\
\hline GM-CSF & 188.57 & $13.69-2597.52$ & 0.003 & $0.0001-0.09$ \\
\hline MCP-1 & 7.09 & $2.91-17.28$ & 17.99 & $6.81-47.49$ \\
\hline IL-8 & 7.31 & $3.75-14.25$ & 3.80 & $1.47-9.81$ \\
\hline Anti-spike IgG antibody & 6.66 & $1.68-26.37$ & 16.340 & $6.72-39.74$ \\
\hline $\mathrm{MIP} 1 \alpha$ & 10.88 & $4.56-25.94$ & & \\
\hline IL-15 & 9.99 & $4.08-24.08$ & & \\
\hline IL-5 & 5.43 & $1.99-14.83$ & & \\
\hline IL-1RA & 3.00 & $1.26-7.13$ & & \\
\hline TNF $\alpha$ & 2.68 & $1.45-4.96$ & & \\
\hline IL-6 & 2.38 & $1.46-3.88$ & & \\
\hline IL-10 & 1.61 & $1.03-2.83$ & & \\
\hline
\end{tabular}

IRR, Incidence Rate Ratio; IRRa, Incidence Rate Ratio adjusted.

significant positive correlation has been reported between COVID-19 infections, mortality and the prevalence of obesity (12). Indeed, previous studies in other Latin cohorts such as a Mexican one (13) have described obesity as the strongest predictor for severe COVID-19, followed by the presence of diabetes and hypertension. However, we found no differences among groups for diabetes and hypertension. This is of relevance given that the prevalence of obesity in Peru is close to 20\% (12). Obesity confers chronic inflammation that can modify innate and adaptive immune responses, resulting in a less responsive immune system and greater vulnerability to infection (14).

We found that lymphocyte concentrations were lower in patients with a worse prognosis, being significantly associated with death and suggesting that lymphopenia correlates with the severity of infection. Indeed, it has been proposed that lymphocyte values are a reliable predictor of clinical outcomes in patients with COVID-19, and may be useful to establish prognosis in the clinical management of patients with this disease $(15,16)$. Furthermore, differences in CRP, D-dimer and creatinine levels had been previously described among groups with different severity, with increased D-dimer levels being associated with worse clinical outcomes, and increased CRP levels with disease progression in COVID-19 patients $(5,17)$.

Cytokine and chemokine levels can considerably vary during the course of COVID-19 based on the phase of the disease, the administration of immunomodulatory medications or the intrinsic characteristics of the patients (18). We found that at baseline and during hospitalization, some immune markers were predictive of subsequent clinical progression, and could, therefore, identify patients at risk of death. In our analysis we observed that several biomarkers, including GM-CSF, MCP-1, MIP1 $\alpha$, TNF $\alpha$, IL-1RA, IL-6, IL-8, IL-15 and IL-10 were associated with fatal outcomes in COVID-19. However, our multivariable analysis showed GM-CSF, MCP-1, and IL-15, IL8 as independent biomarkers associated with fatal outcomes.

The finding of markedly increased monocyte/macrophage activation markers such as MIP- $1 \alpha$ and MCP-1 in COVID-19 patients is similar to a previous study describing elevated MIP$1 \alpha$ levels in all COVID-19 patients and elevated MCP-1 values in patients who died (19). In addition, IP-10 and MCP-3 levels have been shown to be highly associated with disease severity and predict the progression (20). The NF- $\kappa \mathrm{B}$ dependent proinflammatory biomarkers, IL- 6 and TNF $\alpha$, were increased in both the severe and deceased patient groups, similarly to previous reports $(19,20)$. Both cytokines were also described in response to spike protein stimulation in SARS-coronavirus (21). 

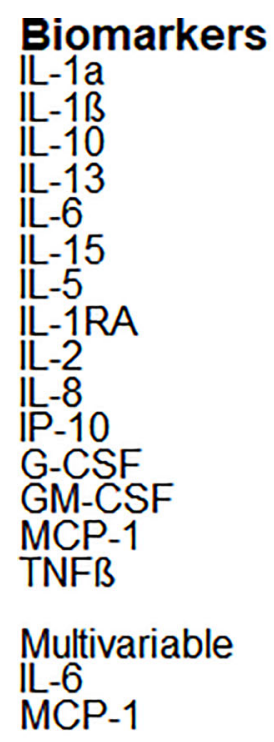
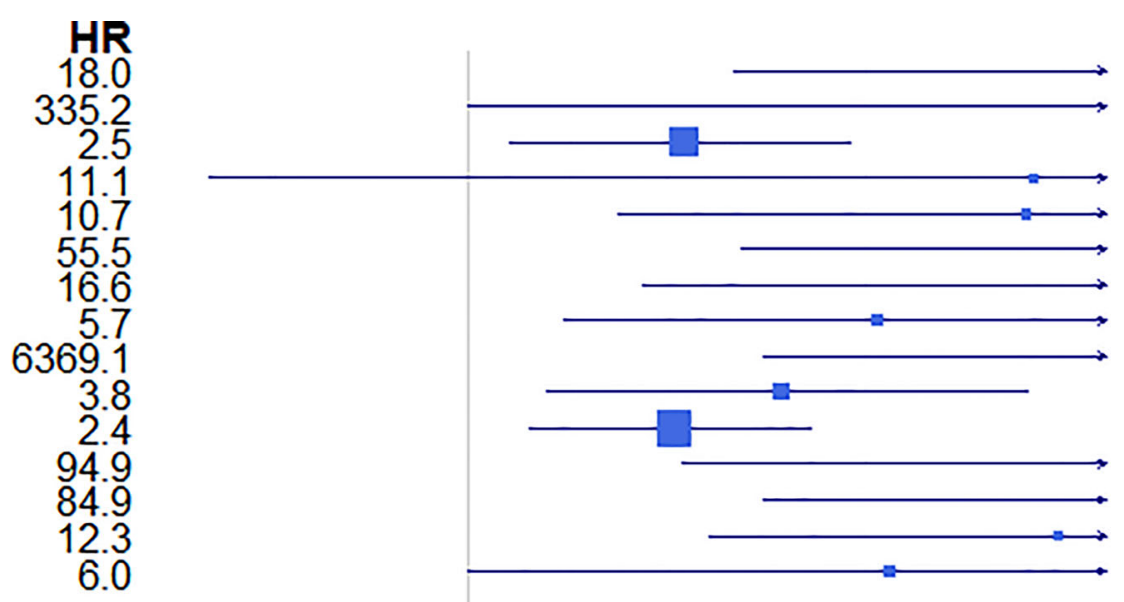

6.8
4.6

FIGURE 6 | Estimates of the magnitude of association (hazard ratios -HR-) and their confidence intervals between biomarkers and the outcome "death". HR were computed by univariable and multivariable Cox models.

Early and persistent expression of proinflammatory cytokines culminates in systemic hyperinflammation associated with the sudden development of respiratory failure requiring hospitalization and often admission to the ICU (7). Regarding IL-6, some reports have only found a significant association in later stages of the disease and mortality $(22,23)$. However, in our study, significantly higher levels were reported from hospital admission, and were predictive of disease progression. Despite the association of these biomarkers in patients with greater severity, higher IL-6 and IL-8 levels were also observed with non-COVID-ARDS (24). IL-8 is considered an important chemotactic factor, involving neutrophil activation and recruitment, associated with COVID-19 prognosis, and has previously been proposed as a biomarker of ARDS (25). Moreover, IL- 8 activates neutrophils as demonstrated by higher counts in deceased COVID-19 patients. Similar to IL-8, we found IL-15 at baseline and longitudinally to be associated with mortality. IL-15 is involved in inflammatory responses, promoting the production and secretion of IL- 8 by neutrophils, which contribute to migration and recruitment at inflammatory sites (26). Furthermore, IL-10 amplifies viral sepsis-related hyperinflammation in critically ill and severe COVID-19 patients and is linked to T-cell exhaustion, presumably through overactivation and proliferation (27). Previously, higher expression of pro-inflammatory cytokine levels (IFN- $\alpha$, TNF, IL-6, IL-8, IL-10, and IP-10) were described in monocytederived macrophages in SARS-CoV infections (28). Among these, IP-10, IL-10 and IL-6 have been proposed as a triad predictive of subsequent COVID-19 clinical progression (5).

In addition, IL- 6 and MCP-1 have also been proposed as candidate markers for disease prediction in hospitalized patients with respiratory failure (29), and in the regression analysis we found IL-6 and MCP-1 in hospitalized patients to be the main predictors of death. MCP-1 levels suggest that secretion is associated with lung injury in severe COVID-19 (30) playing a pathogenic role in respiratory failure during hospitalization (29).

In relation to growth factors, we found that G-CSF and GMCSF levels were related to disease severity. These biomarkers are associated with inflammatory conditions and promote cytokine production in macrophages in the presence of inflammation, perpetuating the inflammatory process in patients with a poor prognosis (31). In our study in both baseline and longitudinal samples, GM-CSF was identified as a biomarker of fatal outcome, although in a previous study no significant differences in severe and moderate disease groups were described (22).

Furthermore, IL-1RA, a negative regulator of IL-1 $\beta$, was also associated with fatal outcomes, as identified previously in another study in the first week of hospitalization (22).

Our results revealed that serum levels of the interferon -inducible protein, IP-10, were also associated with a worse prognosis, and it has been suggested that IP-10 may be a good biomarker for predicting disease progression (5, 32). IP-10 exhibits pro-inflammatory and anti-angiogenic properties and has been proposed as a link between inflammation and angiogenesis that affect COVID-19 patients $(33,34)$. The IP-10 is correlated with IFN type I response. Thus, it makes sense that we found lower concentrations of IFN $\alpha$ in the severe group. Specifically, IFN $\alpha$ is a biomarker mainly produced by virusinfected leukocytes. IFN stimulates the synthesis of major histocompatibility complex class 1 proteins, which are involved in the presentation of viral antigens for recognition by the immune system. The absence of IFN type I expression may impact the ability of dendritic cells to regulate immunity, leading to inadequate priming of antiviral response by CD4 and CD8 T 
cells (35). SARS-CoV-2 infection triggers proinflammatory response long before IFN-mediated antiviral defenses are induced, if at all. This would explain the long incubation time of the virus and long persistence in the respiratory tract, which may be attributed to delayed and/or reduced production of IFN types I and III (7).

It has been suggested that some cytokine patterns associated with COVID-19 severity, such as M-CSF, IP-10 and IL-1RA, are associated with the presence of the macrophage activation syndrome (36) in which infection of macrophages and dendritic cells potentially plays a major role in COVID-19 pathogenesis, even in the absence of productive infection (28).

Of note, there is a tendency for the values of some biomarkers to be higher in the moderate group than in the severe group (GM-CSF, MCP-1, IL-15, TNF $\alpha$, IL-1RA, IFN $\alpha$ ), which could be due to a delay at the time of hospitalization, due to an overloaded health system in the first wave of COVID-19 in Peru, or due to a delay in taking the sample that favors this tendency.

We found no significant differences in RANTES (CCL5) levels. However, a previous study reported that RANTES was significantly elevated in early stages in moderately but not in severely ill patients (22). We observed a trend to low concentrations in the deceased group and a negative correlation with pro-inflammatory and anti-inflammatory biomarkers, indicating that this biomarker may be related to less severe disease conditions. Apart from cytokines and chemokines, antiSARS-CoV-2 antibodies play a role in the severity of COVID-19. It has been reported that SARS-CoV-2 antibody levels vary according to sex, blood type, and also severity and mortality (37). The vast majority of patients in this study (87.3\%) had detectable anti-S1 IgG levels in the first days after admission. Additionally, higher anti-S1 IgG levels were associated with fatal outcomes. One of the previous studies reporting also higher titers of anti-SARS-CoV-2 antibodies in severe COVID-19 compared to individuals with mild disease also found lower viral loads in severe cases (38). High titers of anti-SARS-CoV-2 IgG in severe patients could activate signaling circuits that dampen cellular responses to interferons by blocking the expression of interferon-stimulated genes associated with milder forms of the disease (38). It has been proposed that this exaggerated response pits the immune system against itself (38). From the point of view of clinical applicability, it is important to highlight that it has been proposed that anti-SARSCoV-2 IgG can predict improvement better than commonly used clinical parameters such as lymphocyte and neutrophil counts, and ferritin, D-dimer levels (5).

Regarding to the relationship between antibodies and cytokines, anti-spike 1 IgG antibodies presented a positive correlation with EGF and IL-8 and an inverse correlation with IP-10, IFN- $\gamma$ and G-CSF. A previous study only found a correlation with IL-12p70/IL33 and IgG seroconversion, which correlated with disease severity (39). However, another study hypothesized that IL- 8 and overall, the inflammation acts as a mediator between the association of IgG antibodies and the clinical outcome (40).

The limitations of this study include a relatively low sample size $(n=55)$ and potential false positives as multiple biomarkers and tests were performed without adjusting for multiple testing. Therefore, results should be interpreted with prudence and require further validation but are consistent with published studies in other populations and compatible with the current knowledge of COVID-19 severity, so findings remain relevant. Another limitation is the lack of information about the time period from symptoms onset or positive PCR to admission, which could affect biomarker concentrations. However, our study was carried out in the real-life conditions of COVID-19 cases management at most hospitals in Peru.

Besides stratification of patients at hospitalization, identifying biomarkers associated with mortality in COVID-19 would help to develop new therapeutic approaches and avoid poor outcomes. Immunomodulatory agents could considerably change the expression of these biomarkers. For example, antiinflammatory drug, dexamethasone has been used in the treatment to COVID-19 as an immunomodulatory agent targeting the IFN-mediated signaling, apart from inflammation (24). At hospital admission, corticosteroids were administered to all patients in Peru and, thus, we could not evaluate its role in the prognosis of COVID-19. Nonetheless, previous studies have reported that its use is not associated with clear benefits in mortality (19), although it has been shown to lower mortality in patients receiving either invasive mechanical ventilation or oxygen alone, but not among those not receiving respiratory support (41). The monoclonal antibody against the IL-6 receptor, tocilizumab, proposed for the treatment of COVID patients was not administered (42) since it was demonstrated not to be effective for preventing intubation or death in moderately ill hospitalized patients with COVID-19 (43).

Finally, it is important to acknowledge that several studies have explored the value of assessing cytokines as predictors of death, finding different results. The various quantification methods, kits, relatively low sample sizes in many studies and populations explored might explain these differences, making evident that further studies should be performed to validate biomarkers.

In conclusion, in this Peruvian cohort, various biomarkers of COVID-19 prognosis and fatal outcome, have been identified, validating previous results in other populations involving the same markers. These results should be further explored to identify novel criteria for COVID-19 patient stratification at hospital admission and potential targets for drug development.

\section{DATA AVAILABILITY STATEMENT}

The raw data supporting the conclusions of this article will be made available by the authors, without undue reservation.

\section{ETHICS STATEMENT}

The studies involving human participants were reviewed and approved by Comité de Ética en Investigación para COVID-19 del Seguro Social de Salud - Essalud. Written informed consent 
for participation was not required for this study in accordance with the national legislation and the institutional requirements.

\section{AUTHOR CONTRIBUTIONS}

MP, MU, and GM conceived the study. MP, AM-H, BY, and ST performed assay serum antibodies and cytokines, YS and LA participated in the analysis of IgG titters. MU coordinated patient cohort enrolment and clinical data collection. RG and $\mathrm{AB}$ participated in sample collection. MP and GM carried out the statistical analyses. MP wrote the manuscript. MU and MP supervised the project. All authors contributed to the article and approved the submitted version.

\section{REFERENCES}

1. Lai CC, Shih TP, Ko WC, Tang HJ, Hsueh PR. Severe Acute Respiratory Syndrome Coronavirus 2 (SARS-CoV-2) and Coronavirus Disease.2019 (COVID-19): The Epidemic and the Challenges. Int J Antimicrobial Agents (2020) 55(3):105924. doi: 10.1016/j.ijantimicag.2020.105924

2. Wu Z, McGoogan JM. Characteristics of and Important Lessons From the Coronavirus Disease 2019 (COVID-19) Outbreak in China: Summary of a Report of 72314 Cases From the Chinese Center for Disease Control and Prevention. JAMA (2020) 323(13):1239.1242. doi: 10.1001/ jama.2020.2648

3. Olejnik J, Hume AJ, Mühlberger E. Toll.like Receptor 4 in Acute Viral Infection: Too Much of a Good Thing. PLoS Pathog (2018) 14(12): e1007390. doi: 10.1371/journal.ppat.1007390

4. Coperchini F, Chiovato L, Croce L, Magri F, Rotondi M. The Cytokine Storm in COVID-19: An Overview of the Involvement of the Chemokine/ Chemokine.Receptor System. Cytokine Growth Factor Rev (2020) 53:25-32. doi: 10.1016/j.cytogfr.2020.05.003

5. Laing AG, Lorenc A, Del Molino Del Barrio I, Das A, Fish M, Monin L, et al. A Dynamic COVID-19 Immune Signature Includes Associations With Poor Prognosis. Nat Med (2020) 26(10):1623-35. doi: 10.1038/ s41591.020.1038.6

6. Remy KE, Mazer M, Striker DA, Ellebedy AH, Walton AH, Unsinger J, et al. Hotchkiss RS2. Severe Immunosuppression and Not a Cytokine Storm Characterizes COVID-19 Infections. JCI Insight (2020) 5(17):e140329. doi: $10.1172 /$ jci.insight. 140329

7. Galani IE, Rovina N, Lampropoulou V, Triantafyllia V, Manioudaki M, Pavlos E, et al. Untuned Antiviral Immunity in COVID-19 Revealed by Temporal Type I/III Interferon Patterns and Flu Comparison. Nat Immunol (2021) 22:32-40. doi: 10.1038/s41590.020.00840.x

8. Mahmoudi S, Rezaei M, Mansouri N, Marjani M, Mansouri D. Immunologic Features in Coronavirus Disease 2019: Functional Exhaustion of T Cells and Cytokine Storm. J Clin Immunol (2020) 40(7):974-6. doi: 10.1007/ s10875.020.00824.4

9. Ye Q, Wang B, Mao J. The Pathogenesis and Treatment of the 'Cytokine Storm' in COVID-19. J Infect (2020) 80(6):607-13. doi: 10.1016/ j.jinf.2020.03.037

10. Neumann J, Prezzemolo T, Vanderbeke L, Roca CP, Gerbaux M, Janssens S, et al. An Open Resource for T Cell Phenotype Changes in COVID-19 Identifies $\mathrm{IL}^{*} 10$-producing Regulatory $\mathrm{T}$ Cells as 344 Characteristic of Severe Cases. Clin Transl Immunology (2020) 13;9(11):e1204. doi: 10.1002/ cti2.1204

11. Richardson S, et al. Presenting Characteristics, Comorbidities, and Outcomes. Among 5700 Patients Hospitalized With COVID-19 in the New York City Area. JAMA (2020) 40(7):974-6. doi: 10.1001/jama.2020.6775

12. Jayawardena R, Jeyakumar DT, Misra A, Hills AP, Ranasinghe P. Obesity: A Potential Risk Factor for Infection and Mortality in the Current COVID-19 Epidemic. Diabetes Metab Syndr Nov Dec (2020) 14(6):2199-203. doi: 10.1016/j.dsx.2020.11.001

\section{FUNDING}

This work was supported by funds from "Kaelin Prize 2020" from EsSalud and internal funds of Universidad Cientifica del Sur.

\section{ACKNOWLEDGMENTS}

We would like to thank the laboratory staff of the Hospital Nacional Guillermo Almenara Irigoyen and the research laboratory of the Universidad Cientifica del Sur for their support in the effort to carry out this project in the midst of the COVID-19 wave in Lima, Peru.

13. Hernández.Garduño E. Obesity is the Comorbidity More Strongly Associated for COVID-19 in Mexico. A Case-Control Study. Obes Res Clin Pract (2020) 14(4):375-9. doi: 10.1016/j.orcp.2020.06.001

14. Dhurandhar NV, Bailey D, Thomas D. Interaction of Obesity and Infections. Obes Rev (2015) 16(12):1017-102 doi: 10.1111/obr.12320

15. Ponti G, Maccaferri M, Ruini C, Tomasi A, Ozbend T. Biomarkers Associated With COVID-19 Disease Progression. Crit Rev Clin Lab Sci (2020) 57(6):38999. doi: 10.1080/10408363.2020.1770685

16. Lee J, Park SS, Kim TY, Lee DG, Kim DW. Lymphopenia as a Biological Predictor of Outcomes in COVID-19 Patients: A Nationwide Cohort Study. Cancers (Basel) (2021) 13(3):471. doi: 10.3390/cancers13030471

17. Bikdeli B, Madhavan MV, Jimenez D, Chuich T, Dreyfus I, Driggin E. COVID-19 and Thrombotic or Thromboembolic Disease: Implications for Prevention, Antithrombotic Therapy, and Follow-Up: JACC State-of-the-Art Review. J Am Coll Cardiol (2020) 75(23):2950-73. doi: 10.1016/ j.jacc.2020.04.031. Apr 15 pii:S0735.1097(20)35008.7.

18. Lucas C, Wong P, Klein J, Castro TBR, Silva J, Sundaram M, et al. Longitudinal Analyses Reveal Immunological Misfiring in Severe COVID-19. Nature (2020) 584(7821):463-9. doi: 10.1038/s41586-020-2588-y

19. Abers MS, Delmonte OM, Ricotta EE, Fintzi J, Fink DL, Almeida de Jesus AA, et al. An Immune.Based Biomarker Signature is Associated With Mortality in COVID-19 Patients. JCI Insight (2020) 6(1):e144455. doi: 10.1172/ jci.insight.144455

20. Yang Y, Shen C, Li J, Yuan J, Wei J, Huang F, et al. Plasma IP-10 and MCP-3 Levels Are Highly Associated With Disease Severity and Predict the Progression of COVID-19. J Allergy Clin Immunol (2020) 146(1).119-27.e4. doi: 10.1016/j.jaci.2020.04.027

21. Wang W, Ye L, Ye L, Li B, Gao B, Zeng Y, et al. Up.regulation of IL.6 and TNF-alpha Induced by SARS-Coronavirus Spike Protein in Murine Macrophages via NF-kappaB Pathway. Virus Res (2007) 128(1.2):1.8. doi: 10.1016/j.virusres.2007.02.007

22. Zhao Y, Qin L, Zhang P, Li K, Liang L, Sun J, et al. Longitudinal COVID-19 Profiling Associates IL-1RA and IL-10 With Disease Severity and RANTES With Mild Disease. JCI Insight (2020) 5(13):e139834. doi: 10.1172/ jci.insight.139834

23. Avila.Nava A, Cortes.Telles A, Torres.Erazo D, López.Romero S, Chim Aké R, Ana Gutiérrez Solis L. Serum IL-6: A Potential Biomarker of Mortality Among SARS-CoV-2 Infected Patients in Mexico. Cytokine (2021) 143:155543. doi: 10.1016/j.cyto.2021.155543

24. Sarma A, Christenson S, Mick E, Deiss T, DeVoe C, Pisco A, et al. COVID-19 ARDS is Characterized by a Dysregulated Host Response That Differs From Cytokine Storm and Is Modified by Dexamethasone. Res Sq (2021) 14:rs.3.rs141578. doi: 10.21203/rs.3.rs.141578/v1.

25. García.Laorden MI, Lorente JA, Flores C, Slutsky AS, Villar J. Biomarkers for the Acute Respiratory Distress Syndrome: How to Make the Diagnosis More Precise. Ann Transl Med (2017) 5(14):283. doi: 10.21037/atm.2017.06.49

26. Cassatella MA. And McDonald PP. Interleukin.15 and its Impact on Neutrophil Function. Curr Opin Hematol (2000) 7(3):174.7. doi: 10.1097/ 00062752-200005000-00008 
27. Li H, Liu L, Zhang D, Xu J, Dai H, Tang N. SARS-CoV-2 and Viral Sepsis: Observations and Hypotheses. Lancet 395(10235):1517-20. doi: 10.1016/ S0140-6736(20)30920-X

28. Zheng J, Wang Y, Li K, Meyerholz DK, Allamargot C, Perlman S. Severe Acute Respiratory Syndrome Coronavirus 2-Induced Immune Activation and Death of Monocyte-Derived Human Macrophages and Dendritic Cells. J Infect Dis (2021) 223(5):785-95. doi: 10.1093/infdis/jiaa753

29. Jøntvedt Jørgensen M, Cato Holter J, Egeland Christensen E, Schjalm C, Tonby K, Pischke SE, et al. Increased Interleukin.6 and Macrophage Chemoattractant Protein.1 Are Associated With Respiratory Failure in COVID-19. Sci Rep (2020) 10:21697. doi: 10.1038/s41598-020-78710-7

30. Mehta P, McAuley DF, Brown M, Sanchez E, Tattersall RS, Manson JJ, et al. COVID-19: Consider Cytokine Storm Syndromes and Immunosuppression. Lancet (2020) 395:1033-4. doi: 10.1016/S0140.6736(20)30628.0

31. Hamilton JA. Colony.stimulating Factors in Inflammation and Autoimmunity. Nat Rev Immunol (2008) 8(7):533-44. doi: 10.1038/nri2356

32. Haroun RA, Osman WH, Eessab AM. Interferon. $\gamma$. Induced Protein 10 (IP.10) and Serum Amyloid A (SAA) are Excellent Biomarkers for the Prediction of COVID-19 Progression and Severity. Life Sci (2021) 269:119019. doi: 10.1016/ j.lfs.2021.119019

33. Gotsch F, Romero R, Friel L, Kusanovic JP, Espinoza J, Erez O, et al. CXCL10/ IP.10: A Missing Link Between Inflammation and Anti-Angiogenesis in Preeclampsia? J Matern Fetal Neonatal Med (2007) 20(11):777-92. doi: 10.1080/14767050701483298

34. Ackermann M, Mentzer SJ, Kolb M, Jonigk D. Inflammation and Intussusceptive Angiogenesis in COVID-19: Everything in and Out of Flow. Eur Respir J (2020) 56(5):2003147. doi: 10.1183/13993003.03147.2020

35. Montoya M, Schiavoni G, Mattei F, Gresser I, Belardelli F, Borrow P, et al. Type I Interferons Produced by Dendritic Cells Promote Their Phenotypic and Functional Activation. Blood (2002) 99(9):3263.71. doi: 10.1182/blood.v99.9.3263

36. Huntington KE, Louie AD, Lee CG, Elias JA, Ross EA, El.Deiry WS. Cytokine Ranking via Mutual Information Algorithm Correlates Cytokine Profiles With Presenting Disease Severity in Patients With COVID-19. Elife (2021) 10: e64958. doi: 10.7554/eLife.64958.

37. Horspool AM, Kieffer T, Russ BP, DeJong MA, Wolf MA, Karakiozis JM, et al. Interplay of Antibody and Cytokine Production Reveals CXCL13 as a Potential Novel Biomarker of Lethal SARS-CoV-2 Infection. mSphere (2021) 6(1):e01324.20. doi: 10.1128/mSphere.01324.20
38. Combes AJ, Courau T, Kuhn NF, Hu KH, Ray A, Chen WS, et al. Global Absence and Targeting of Protective Immune States in Severe COVID-19. Nature (2021) 591(7848):124.130. doi: 10.1038/s41586.021.03234.7

39. Munitz A, Edry.Botzer L, Itan M, Tur.Kaspa R, Dicker D, Marcoviciu D, et al. Rapid Seroconversion and Persistent Functional IgG Antibodies in Severe COVID-19 Patients Correlates With an IL.12p70 and IL-33 Signature. Sci Rep (2021) 11:3461. doi: 10.1038/s41598-021-83019-0

40. Liu L, Chen HG, Li Y, Li H, Li J, Wang Y, et al. Temporal Profiles of Antibody Responses, Cytokines, and Survival of COVID-19 Patients: A Retrospective Cohort in Wuhan, China. Eng (Beijing) (2021) 18. doi: 10.1016/ j.eng.2021.04.015

41. RECOVERY Collaborative Group, Horby P, Emberson JR, Mafham M, Bell JL. Dexamethasone in Hospitalized Patients With COVID-19. N Engl J Med (2021) 384:693.704. doi: 10.1056/NEJMoa2021436

42. King A, Vail A, O'Leary C, Hannan C, Brough D, Patel H, et al. Anakinra in COVID-19: Important Considerations for Clinical Trials. Lancet Rheumatol (2020) 2:e379-81. doi: 10.1016/S2665-9913(20)30160-0

43. Stone JH, Frigault MJ, Serling-Boyd NJ, Fernandes AD, Harvey L, et al. Efficacy of Tocilizumab in Patients Hospitalized With COVID-19. N Engl J Med (2020) 383:2333.2344. doi: 10.1056/NEJMoa2028836

Conflict of Interest: The authors declare that the research was conducted in the absence of any commercial or financial relationships that could be construed as a potential conflict of interest.

Publisher's Note: All claims expressed in this article are solely those of the authors and do not necessarily represent those of their affiliated organizations, or those of the publisher, the editors and the reviewers. Any product that may be evaluated in this article, or claim that may be made by its manufacturer, is not guaranteed or endorsed by the publisher.

Copyright ( 2021 Pons, Ymaña, Mayanga-Herrera, Sáenz, Alvarez-Erviti, Tapia-Rojas, Gamarra, Blanco, Moncunill and Ugarte-Gil. This is an open-access article distributed under the terms of the Creative Commons Attribution License (CC BY). The use, distribution or reproduction in other forums is permitted, provided the original author(s) and the copyright owner(s) are credited and that the original publication in this journal is cited, in accordance with accepted academic practice. No use, distribution or reproduction is permitted which does not comply with these terms. 